\title{
Intraoperative Finding of Hepatic Hydatid Cyst with Fine Needle Aspiration: A Case Study with AFB and GMS Special Stains Highlighting the Echinococcal Hooklets
}

\author{
Ozlem Fidan-Ozbilgin ${ }^{*}$, Stefan E Pambuccian, Swati Mehrotra and Güliz A Barkan
}

Department of Pathology, Loyola University Medical Center, USA

"Corresponding author: Fidan-Ozbilgin O, Department of Pathology, Loyola University Medical Center, 2160 S 1st Ave Maywood, IL 60153, USA, Tel: 708-327-2081; Fax: 708-216-8225; E-mail: ozlemfo@gmail.com, GBARKAN@lumc.edu

Received date: May 16, 2016; Accepted date: July 20, 2016; Published date: August 01, 2016

Copyright: (c) 2016 Fidan-Ozbilgin O, et al. This is an open-access article distributed under the terms of the Creative Commons Attribution License, which permits unrestricted use, distribution, and reproduction in any medium, provided the original author and source are credited.

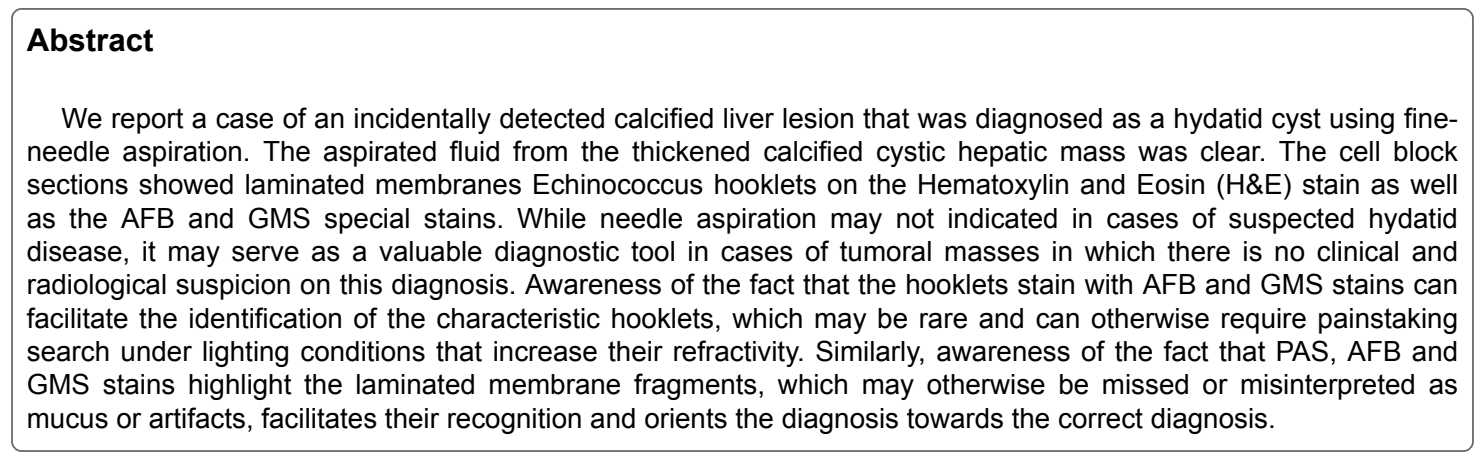

Keywords: Echinococcosis; Liver; Cytology; FNA; GMS; AFB; Hooklets

\section{Introduction}

Hydatid cyst disease is a zoonotic infection that results from tissue infestation with the larval stage of the parasite Echinococcus granulosus [1]. The disease is endemic in sheep-raising regions of the world, especially in Mediterranean countries, the Middle East, Eastern Europe, South America, Australia and New Zealand. While still uncommon in the United States, travel and changes in immigration patterns over the past four decades have caused a rise in the number of cases hydatid disease throughout North America. This has led to the need for increased awareness of its clinical features, diagnosis, and management. Fine needle aspiration (FNA) cytology is typically not performed in cases with a suspicion of hydatid cyst due to the perceived risk of anaphylactic shock [2], which makes it difficult for cytopathologists to gain experience with such cases. Cytologically, protoscolices, hooklets, and fragments of the laminated membrane are commonly found in hydatid cysts [3-5].

\section{Case Report}

A 67-year-old Hispanic woman presented to the emergency room complaining of two week long left lower quadrant abdominal pain and recent-onset hematochezia. Her past medical history included a benign ovarian cyst and diverticulosis. Physical examination was unremarkable; no abdominal mass was palpated. Routine blood count, biochemistry and urine analysis were within normal limits. Serologic testing for echinococcosis was not performed. Her abdominal computed tomography $(\mathrm{CT})$ scan revealed a $4.5 \mathrm{~cm}$ multi-laminated calcification in the inferior right lobe of the liver, in addition to cholelithiasis. The liver lesion was interpreted radiologically as "likely scar from old inflammatory lesion" (Figure 1).

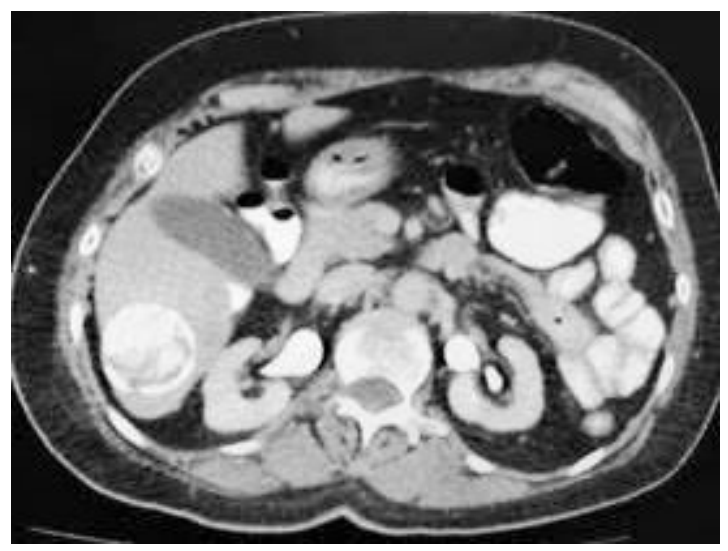

Figure 1: Computed tomography of the abdomen showing a multilaminated calcification in the right hepatic lobe.

During the laparoscopic cholecystectomy, an intraoperative cholangiogram, and FNA of the liver lesion were performed. Several passes targeting the calcified mass on the lateral edge of the right lobe were performed using a percutaneous Tru-Cut needle. The aspirated clear fluid was submitted in formalin for cytologic examination. No microbiologic examination was performed. No allergic reaction or other complication was observed following the FNA. The fluid was centrifuged and a cell block was prepared from the sediment using the thrombin technique. The cell block sections were stained with hematoxylin and eosin (H\&E), PAS, GMS, and AFB stains. 
Citation: Fidan-Ozbilgin O, Pambuccian SE, Mehrotra S, Barkan GA (2016) Intraoperative Finding of Hepatic Hydatid Cyst with Fine Needle Aspiration: A Case Study with AFB and GMS Special Stains Highlighting the Echinococcal Hooklets. J Cytol Histol 7: 422. doi: 10.4172/2157-7099.1000422

Page 2 of 4

Microscopic examination of the H\&E-stained cell block sections revealed fragments of the laminated membrane. These appeared as acellular material with delicate undulating parallel striations in the background of amorphous necrotic debris and fine granular calcifications ("calcareous bodies"). No inflammatory cells were seen. In addition, upon lowering the condenser, we could appreciate detached hooklets that appeared as scimitar-shaped refractile structures (Figure 2).
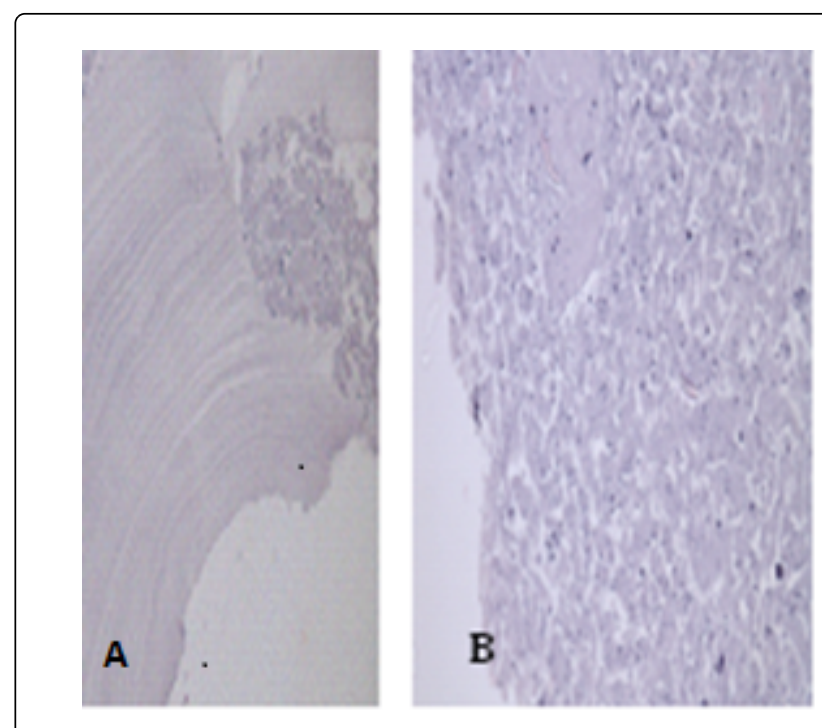

Figure 2: (H\&E, low power) Cytologic findings. (A) Acellular laminated membrane in the background of necrotic debris. (B) Detached hooklets with refractile structures in the necrotic and fine calcified debris.

These structures were transparent and therefore not easily seen on H\&E-stained sections, but were highlighted by GMS and AFB special stains, which also stained the laminated membrane fragments (Figure 3).
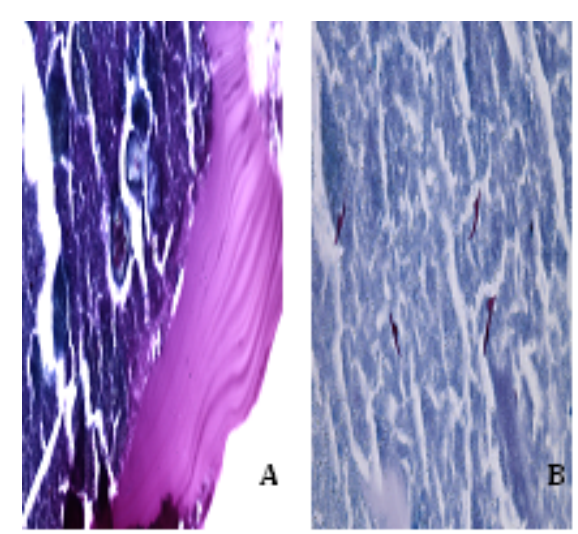

Figure 3: Special stain findings. (Acid Fast Stain, high power) (A) Laminated membrane and hooklets (B) appear prominent and pink-red on AFB special stain. The laminated membrane fragments also stained for PAS.
Based on these cytomorphological findings, a diagnosis of hydatid cyst was made (Figure 4).
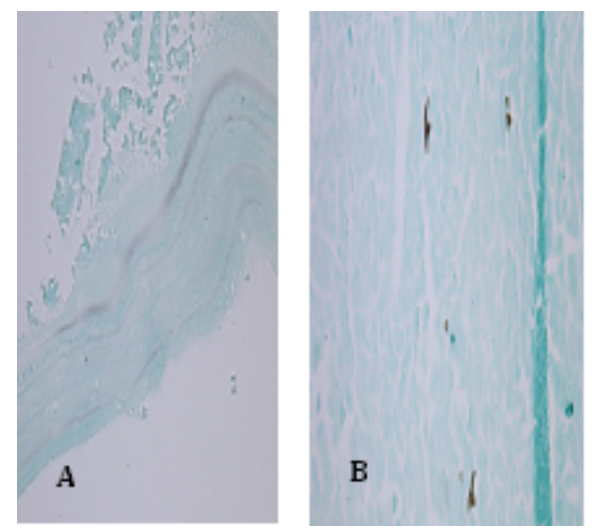

Figure 4: Special stain findings. (Gomori Methanamine Silver Stain, high power) Laminated membrane (A) and hooklets (B) appear prominent and black on GMS special stain.

\section{Discussion}

Hydatid disease or human cystic echinococcosis is a parasitic infestation caused in the majority of cases by Echinococcus granulosus and multilocularis, the latter causing alveolar echinococcosis, characterized by the presence of multiple small cysts. Dogs and other canidae are the parasite's definite host, while sheep and goats, and occasionally other warm-blooded vertebrates serve as the parasite's intermediate hosts. Humans are accidental intermediate hosts and become infected by ingestion of raw fruits and vegetables contaminated with eggs shed by dogs or foxes. After ingestion, the eggs of E. granulosus hatch, and the larval oncospheres pass to the liver by the portal vein. The cysts most often occur in the liver $(60-70 \%$ of cases), predominantly affecting the right lobe, although they may be multiple and involve all lobes of the liver. The second most frequently involved organ is the lung (20-25\% of cases), but hydatid cysts can occur anywhere in the body, including in unusual locations such as bones, kidneys, spleen, muscles, CNS and orbit.

The adult E. granulosus is a small tapeworm that measures 3 to 5 $\mathrm{mm}$ in length and shows a scolex (head) with four suckers and a double crown of 28-50 hooks. The cysts, which can grow up to 10-20 $\mathrm{cm}$, are composed of three layers[6]: an (1) outer adventitial layer (pericyst or ectocyst) consisting of dense fibrous tissue predominantly of host tissue derivation, (2) the middle $1 \mathrm{~mm}$ thick avascular, eosinophilic, refractile and anucleated laminated membrane and (3) an inner $10-25 \mu \mathrm{m}$ thick cellular germinal layer. The cysts contain abundant clear fluid into which the daughter cysts ("brood capsules") formed by the germinal layer are released. Each of these daughter cysts contains scolices with numerous hooklets. The hydatid cysts grow slowly and ultimately the parasite dies, and the cysts degenerate and calcify; scolices may be lost in longstanding cysts with degeneration, but the hooklets and calcareous corpuscles persist, and can serve as diagnostic clues on FNA. Unless the parasite dies or the cyst ruptures, the host reaction to the cyst is minimal, which most likely explains why most patients with hydatid cysts are asymptomatic until the cysts attain a large size. Because of their slow growth, the diagnosis may be delayed for months to many years after the initial infection. The 
presence and type of clinical manifestations depends on the cyst's location and size. If the cyst is located in the liver it can cause hepatomegaly, obstructive jaundice, and cholangitis. In other locations, the symptoms are nonspecific, and the disease is rarely suspected outside endemic areas.

Hydatid disease can be diagnosed by serology and imaging studies, but these techniques have low sensitivity and specificity and are therefore not definitive. On imaging studies the cysts may have one of four appearances [7]: unilocular simple cysts (type I), complex cysts with daughter cysts or detached floating membranes ("water lily sign") (type II), calcified cysts (type III) and complicated cysts with rupture or superimposed infection (type IV).

The hydatid cyst can be diagnosed preoperatively or intraoperatively through cyst aspiration by demonstrating characteristic findings of fragments of hyaline, laminated cyst wall membrane and confirmatory findings of scolices or hooklets in the smears. FNA is generally not recommended in suspected cases of hydatid cyst due to the possibility of allergic and even acute anaphylactic reaction. However, this appears to be more of a historical complication, which may have been due to the use of large-bore needles. Complications of needle aspiration, such as minor allergic [8] or major anaphylactic reactions [5] have only rarely been reported, usually when large needles (18G and below) were employed [5]; in most cases of fine needle (21G and above) aspiration of a clinically unsuspected hydatid cyst no complications were encountered $[9,10]$. No complications of needle aspiration were noted in our case either.

Although the majority of hydatid cysts occur in the liver, cases diagnosed by aspiration cytology are more likely to represent extrahepatic disease, such as lung involvement [11] or involvement of unusual sites like the breast $[12,13]$, head and neck locations $[1,14,15]$, soft tissues [16,17], probably reflecting the fact that hydatid cysts were not suspected in these cases. Hydatid cyst should be considered when fragments suggestive of a laminated membrane are present on the cytologic preparations without evidence of protoscolices [10]. A definite diagnosis of hydatid cyst is confirmed by identification of protoscolices or the refractile hooklets. Protoscolices [18,19] are only seen with live organisms; more commonly in cases in which aspiration cytology is performed because hydatid serology is negative, the aspirates show only polygonal fragments of the acellular laminated membrane [4,17], rare scattered hooklets or both fragments of laminated membrane and hooklets $[11,20]$. The background of such aspirates show variable amounts of inflammatory cells (histiocytes, mixed inflammatory cells including neutrophils, eosinophils, giant cells, and rarely granulomas), and granular and calcific debris. However, the aspirate may not show any of the characteristic findings of hydatid cysts; awareness of the pathologist that a hydatid cyst is suspected based on clinical and/or imaging findings may increase their likelihood of diagnosing this condition, by diligently searching for the characteristic cytologic findings [8].

In our case, acellular laminated membrane fragments and refractile hooklets were identified on H\&E stained cell block sections. The special stains GMS and AFB highlighted the hooklets in black and pink respectively. The cyst membrane takes up the periodic acid Schiff (PAS) stain while hooklets are better stained by a Ziehl Neelsen stain [21]. Several other stains such as Ryan trichrome blue stain, and modified Baxby stain can be used in fresh unfixed cyst fluid samples to improve the visualization of hooklets and scolices [22], but are rarely used in cytology practice.
Complete surgical removal of the cyst(s) is the main therapeutic option; when the diagnosis is made or suspected preoperatively, a short course of preoperative albendazole treatment is frequently used because it reduces the likelihood of intraoperative parasite seeding and disease recurrence. Medical treatment alone is not effective, but may reduce the size of the cysts and may therefore be used in patients who are poor surgical candidates due to comorbidities, multi-organ involvement and inoperable cyst location. Postoperative medical treatment with benzimidazole derivates (albendazole, mebendazole) is frequently combined with surgery to prevent recurrence [15].

\section{Conclusion}

We report a case of an incidentally detected calcified liver lesion that was diagnosed as a hydatid cyst using fine-needle aspiration. While needle aspiration may not indicated in cases of suspected hydatid disease, it may serve as a valuable diagnostic tool in cases of tumoral masses in which there is no clinical and radiological suspicion on this diagnosis. Awareness of the fact that the hooklets stain with AFB and GMS stains can facilitate the identification of the characteristic hooklets, which may be rare and can otherwise require painstaking search under lighting conditions that increase their refractivity. Similarly, awareness of the fact that PAS, AFB and GMS stains highlight the laminated membrane fragments, which may otherwise be missed or misinterpreted as mucus or artifacts, facilitates their recognition and orients the diagnosis towards the correct diagnosis.

\section{References}

1. Chakrabarti I, Goswami BK (2012) Primary hydatid cyst of the neck diagnosed by aspiration cytology. Trop Parasitol 2: 127-128.

2. Siracusano A, Delunardo F, Teggi A, Ortona E (2012) Host-parasite relationship in cystic echinococcosis: an evolving story. Clin Dev Immunol.

3. Ciftcioglu MA, Yildirgan MI, Akcay MN, Reis A, Safali M, et al. (1997) Fine needle aspiration biopsy in hepatic Echinococcus multilocularis. Acta Cytol 41: 649-652.

4. Saenz-Santamaria J, Moreno-Casado J, Nunez C (1995) Role of fineneedle biopsy in the diagnosis of hydatid cyst. Diagn Cytopathol 13: 229-232.

5. Agarwal PK, Husain N, Singh BN (1989) Cytologic findings in aspirated hydatid fluid. Acta Cytol 33: 652-654.

6. Golzari SE, Sokouti M (2014) Pericyst: the outermost layer of hydatid cyst. World J Gastroenterol 20: 1377-1378.

7. Polat P, Kantarci M, Alper F, Suma S, Koruyucu MB, et al. (2003) Hydatid disease from head to toe. Radiographics 23: 475-494.

8. von Sinner WN, Nyman R, Linjawi T, Ali AM (1995) Fine needle aspiration biopsy of hydatid cysts. Acta Radiol 36: 168-172.

9. Kim AR, Park SJ, Gu MJ, Choi JH, Kim HJ (2013) Fine Needle Aspiration Cytology of Hepatic Hydatid Cyst: A Case Study. Korean Journal of Pathology 47: 395-398.

10. Hira PR, Shweiki H, Lindberg LG. (1988) Diagnosis of cystic hydatid disease: role of aspiration cytology. Lancet 2: 655-657.

11. Gochhait D, Dey P, Rajwanshi A, Nijhawan R, Radhika S, et al. (2015) Spectrum of fungal and parasitic infections on fine needle aspiration cytology. Diagn Cytopathol 43: 450-455.

12. Geramizadeh B, Boob R, Talei AR, Rasekhi A (2003) Fine needle aspiration cytology in hydatid cyst of the breast. Acta Cytol 47: 701-702.

13. Dhingra KK, Singhal N, Jain S (2006) Unsuspected isolated breast hydatidosis: a cytological diagnosis. Cytopathology 17: 213-215.

14. Daneshbod Y, Khademi B (2009) Hydatid disease of the submandibular gland diagnosed by fine needle aspiration: a case report. Acta Cytol 53: 454-456. 
Citation: Fidan-Ozbilgin O, Pambuccian SE, Mehrotra S, Barkan GA (2016) Intraoperative Finding of Hepatic Hydatid Cyst with Fine Needle Aspiration: A Case Study with AFB and GMS Special Stains Highlighting the Echinococcal Hooklets. J Cytol Histol 7: 422. doi: 10.4172/2157-7099.1000422

Page 4 of 4

15. Goyal P, Ghosh S, Sehgal S (2014) Primary multilocular hydatid cyst of neck with unique presentation: a rare case report and literature review. Head Neck Pathol 8: 334-338.

16. Gupta R, Mathur SR, Agarwala S, Kaushal S, Srivastav A (2008) Primary soft tissue hydatidosis: aspiration cytological diagnosis in two cases. Diagn Cytopathol 36: 884-886.

17. Ascoli V, Teggi A, Gossetti F, Nardi F (1990) Hydatid cyst: primary diagnosis by fine-needle aspiration biopsy. Diagn Cytopathol 6: 44-48.

18. Ingram EA, Helikson MA (1991) Echinococcosis (hydatid disease) in Missouri: diagnosis by fine-needle aspiration of a lung cyst. Diagn Cytopathol 7: 527-531.
19. Parwani AV, Burroughs FH, Ali SZ (2004) Echinococcal cyst of the liver. Diagn Cytopathol 31: 111-112.

20. Das DK, Bhambhani S, Pant CS (1995) Ultrasound guided fine-needle aspiration cytology: diagnosis of hydatid disease of the abdomen and thorax. Diagn Cytopathol 12: 173-176.

21. Handa U, Mohan H, Ahal S (2001) Cytodiagnosis of hydatid disease presenting with Horner's syndrome: a case report. Acta Cytol 45: 784-788.

22. Clavel A, Varea M, Doiz O (1999) Visualization of hydatid elements: comparison of several techniques. J Clin Microbiol 37: 1561-1563. 